\title{
An Intelligent CRM System for Identifying High-Risk Customers: An Ensemble Data Mining Approach
}

\author{
Kin Keung Lai ${ }^{1}$, Lean $\mathrm{Yu}^{1,2}$, Shouyang Wang ${ }^{2}$, and Wei Huang ${ }^{1}$ \\ ${ }^{1}$ Department of Management Sciences, City University of Hong Kong, \\ Tat Chee Avenue, Kowloon, Hong Kong \\ \{mskklai, msyulean\} @cityu. edu.hk \\ ${ }^{2}$ Institute of Systems Science, Academy of Mathematics and Systems Science, \\ Chinese Academy of Sciences, Beijing 100080, China \\ \{yulean, sywang, whuang @ams. .ac.cn
}

\begin{abstract}
In this study, we propose an intelligent customer relationship management (CRM) system that uses support vector machine (SVM) ensembles to help enterprise managers effectively manage customer relationship from a risk avoidance perspective. Different from the classical CRM for retaining and targeting profitable customers, the main focus of our proposed CRM system is to identify high-risk customers for avoiding potential loss. Through experiment analysis, we find that the Bayesian-based SVM ensemble data mining model with diverse components and "choose from space" selection strategy show the best performance over the testing samples.
\end{abstract}

Keywords: Customer relationship management, support vector machine, ensemble data mining, high-risk customer identification.

\section{Introduction}

Customer relationship management (CRM) has become more and more important today due to the intensive competitive environment and increasing rate of change in the customer market. Usually, most enterprises are interested in knowing who will respond, activate, purchase, or use their products or services. However, customer risk avoidance and management is also a critical component to maintain profitability in many industries, such as commercial banks and insurance industries. These businesses are concerned with the amount of risk they are taking by accepting someone or a certain corporate as a customer. Sustainability and profitability of these businesses particularly depends on their ability to distinguish faithful customers from bad ones [1-2]. In order to enable these businesses to take either preventive or correct immediate action, it is imperative to satisfy the need for efficient and reliable model that can accurately identify high-risk customers with potential default trend.

In such a CRM system that focusing on customer risk analysis, a generic approach is to apply a classification technique on similar data of previous customers - both faithful and delinquent customers - in order to find a relationship between the characteristics and potential default [1-2]. One important ingredient needed to accomplish 
this goal is to seek an accurate classifier in order to categorize new customers or existing customers as good or bad. In the process of customer classification, data mining techniques, especially classification techniques, play a critical role. Some existing techniques about risk identification can be referred to [1-3].

Recent studies [2-3] found that the unitary data mining technique did not produce consistently good performance because each data mining technique had their own shortcomings and different suitability. Therefore the ensemble data mining technique is an effective way to remedy this drawback. In this study, our aim is to propose a support vector machine (SVM) based ensemble data mining model to identify the high risk customer. The rest of the study is organized as follows. Section 2 describes a four-step SVM ensemble data mining model for CRM to identify high risk customer in detail. In Section 3, we conduct some experiments with a real-world customer dataset. Finally, some conclusions are drawn in Section 4.

\section{The SVM-Based Ensemble Data Mining System for CRM}

As earlier noted, ensembling multiple classification models into an aggregated output has been an effective way to improve the performance of data mining [2]. A definition of effective ensemble classifiers was introduced by Hansen and Salamon [4], who stated: 'A necessary and sufficient condition for an ensemble of classifiers to be more accurate than any of its individual members is if the classifiers are accurate and diverse.' An accurate classifier is the one that is well trained and whose error rate is better than a random selection of output classes. Two classifiers are diverse if they make different errors on the same input values. Different from their study [5], which is done by using ANN; our tool is SVM, which is a more robust model than ANN. Generally, the SVM-based ensemble data mining system for high risk customers' identification comprises the following four main steps: data preparation, single classifier creation, ensemble member selection, and ensemble classifier construction, which are described as follows.

The first step of this ensemble data mining system is to prepare input data into a readily available format. The main task of this phase is to collect related data and to perform necessary preprocessing. A systematical research about data preparation for complex data analysis is done by Yu et al. [5].

The second step is to create single classifiers. According to the bias-variancecomplexity trade-off principle [6], an ensemble model consisting of diverse individual models (i.e., base models) with much disagreement is more likely to have a good performance. Therefore, how to generate the diverse model is the key to the construction of an effective ensemble model [3]. For the SVM classifier, there are three main ways for generating diverse base models: (a) utilizing different training data sets; (b) changing the kernel functions in SVM, such as polynomial function and Gaussian function; (c) varying the SVM model parameters, such as parameter $C$ and $\sigma^{2}$.

Although there are many ways to create diverse base models, the above approaches are not necessarily independent. In order to guarantee the diversity, a selection step is used to pick out some of them, and construct an ensemble classifier for CRM.

In order to select diverse classifiers, the subsequent step is to select some independent single models. There are many algorithms, such as principal component 
analysis (PCA) [7], choose the best (CTB) [8] and choose from subspace (CFS) [8] for ensemble member selection. The PCA is used to select a subset of members from candidate members using the maximizing eigenvalue of the error matrix. The idea of CTB is to select the classifier with the best accuracy from candidate members to formulate a subset of all members. The CFS is based on the idea that for each model type, it chooses the model exhibiting the best performance. Readers can refer to [7-8].

After single classifiers are selected, ensemble classifier will be combined in the final step. Typically, majority voting based ensemble strategy [4] and Bayesian-based ensemble strategy [11] are the most popular methods for constructing an accurate ensemble model. Interested readers can refer to $[4,11]$ for more details.

\section{Experimental Analysis}

In this section a real-world credit dataset is used to test the performance of the SVMbased ensemble data mining model. The dataset used in this study is from the financial service company of England, obtained from accessory CDROM of a book [12]. For space consideration, the data is omitted. For testing purpose, we randomly divide the scaled dataset into two parts: training set with 800 samples, testing set with 1071 samples. Particularly, the total accuracy [1-2] is used to measure the efficiency of classification. For comparison, logistic regression-based (LogR) ensemble and artificial neural network-based (ANN) ensemble are also conducted. Note that LogR ensemble, ANN ensemble and SVM ensemble are constructed with 20 different classifiers respectively. Accordingly different experimental results are reported in Table 1.

Table 1. The total accuracy of different ensemble models (\%)

\begin{tabular}{cccc}
\hline \multirow{2}{*}{ Ensemble } & Selection & \multicolumn{2}{c}{ Ensemble strategy } \\
\cline { 3 - 4 } & strategy & Majority voting ensemble & Bayesian-based ensemble \\
\hline \multirow{2}{*}{ LogR } & PCA & 57.65 & 60.08 \\
& CTB & 58.63 & 62.29 \\
& CFS & 59.49 & 60.86 \\
ANN & PCA & 68.63 & 73.35 \\
& CTB & 69.06 & 71.24 \\
SVM & CFS & 68.65 & 72.93 \\
& PCA & 70.75 & 76.36 \\
& CTB & 71.30 & 77.68 \\
& CFS & 75.63 & 87.06 \\
\hline
\end{tabular}

From Table 1, we find the following interesting conclusions. (1) Diversity of individual classifiers can improve the performance of the SVM-based ensemble models; (2) CFS always performs the best among the three strategies of ensemble members' selection; (3) Bayesian-based ensemble strategy always performs much better than majority voting ensemble strategy. These results and findings also demonstrate the effectiveness of the SVM-based ensemble models relative to other ensemble approaches, such as logit regression ensemble and neural network ensemble. 


\section{Conclusions}

In this study, we propose an intelligent CRM system that uses SVM ensemble techniques to help enterprise managers effectively manage customer relationship from a risk avoidance perspective. Through experiment analysis, we can easily find that the SVM-based ensemble model performs much better than $\operatorname{LogR}$ ensemble and ANN ensemble, indicating that the SVM-based ensemble models can be used as an effective CRM tool to identify high risk customers.

\section{Acknowledgements}

This work is supported by the grants from the National Natural Science Foundation of China (NSFC No. 70221001, 70601029), Chinese Academy of Sciences (CAS No. 3547600), Academy of Mathematics and Systems Sciences (AMSS No. 3543500) of CAS, and City University of Hong Kong (SRG No. 7001677, 7001806).

\section{References}

1. Lai, K.K., Yu, L., Zhou, L.G., Wang, S.Y.: Credit Risk Evaluation with Least Square Support Vector Machine. Lecture Notes in Computer Science 4062 (2006) 490-495

2. Lai, K.K., Yu, L., Wang, S.Y., Zhou, L.G.: Credit Risk Analysis Using a Reliability-based Neural Network Ensemble Model. Lecture Notes in Computer Science 4132 (2006) 682-690

3. Lai, K.K., Yu, L., Huang, W., Wang, S.Y.: A Novel Support Vector Metamodel for Business Risk Identification. Lecture Notes in Artificial Intelligence 4099 (2006) 980-984

4. Hansen, L., Salamon, P.: Neural Network Ensemble. IEEE Transactions on Pattern Analysis and Machine Intelligence 12 (1990) 993-1001

5. Yu, L., Wang, S.Y., Lai, K.K.: A Integrated Data Preparation Scheme for Neural Network Data Analysis. IEEE Transactions on Knowledge and Data Engineering 18 (2006) 217-230

6. Yu, L., Lai, K.K., Wang, S.Y., Huang, W.: A Bias-Variance-Complexity Trade-Off Framework for Complex System Modeling. Lecture Notes in Computer Science 3980 (2006) 518-527

7. Yu, L., Wang, S.Y., Lai, K.K.: A Novel Nonlinear Ensemble Forecasting Model Incorporating GLAR and ANN for Foreign Exchange Rates. Computers \& Operations Research 32(10) (2005) 2523-2541

8. Partridge D., Yates, W.B.: Engineering Multiversion Neural-Net Systems. Neural Computation 8 (1996) 869-893

9. Vapnik, V.N.: The Nature of Statistical Learning Theory. Springer: New York (1995)

10. Suykens J.A.K., Vandewalle, J.: Least Squares Support Vector Machine Classifiers. Neural Processing Letters 9 (1999) 293-300

11. Xu, L., Krzyzak, A., Suen, C.Y.: Methods of Combining Multiple Classifiers and Their Applications to Handwriting Recognition. IEEE Transactions on Systems, Man, and Cybernetics 22(3) (1992) 418-435

12. Thomas, L.C., Edelman, D.B., Crook, J.N.: Credit Scoring and its Applications. Society of Industrial and Applied Mathematics, Philadelphia (2002) 